\title{
Soft elasticity in smectic elastomers
}

\author{
J. M. Adams and M. Warner \\ Cavendish Laboratory, University of Cambridge, Madingley Road, Cambridge CB3 OHE, United Kingdom
}

(Received 7 March 2005; published 12 July 2005)

\begin{abstract}
We investigate the soft elastic modes of smectic elastomers, that is shape change without energy cost. We use a microscopic model for their nonlinear elasticity, similar to those used for nematic elastomers. We consider two different phases of smectic elastomer; the biaxial smectic A for a simple illustration, and smectic $\mathrm{C}$ phases which are of great practical significance. We show that only one nontrivial trajectory of the director gives soft deformations. We give a geometrical interpretation of this soft elastic mode and give an explicit example.
\end{abstract}

DOI: 10.1103/PhysRevE.72.011703

PACS number(s): 61.30.Vx, 83.80.Va, 62.20.Dc, 61.41.+e

\section{INTRODUCTION}

Soft modes in nematic elastomers can be understood theoretically from both symmetry arguments [1] and from microscopic models [2]. The occurrence of soft modes is independent of the particular description used, since they occur in any model with the same symmetries as a nematic elastomer [3]. Physically, soft modes arise when the distribution of chain shapes is rotated at constant shape (and thus constant entropy and constant nematic energy) while the body changes shape to accommodate the chains [4]. Figure 1 shows the initial chain shape distribution, an intermediate shape where its rotation is not yet complete, and the shape when it has been rotated by $90^{\circ}$ to be along the direction of imposed stretch and shape change at constant energy has come to its end. Such soft modes have been investigated experimentally in both monodomain and polydomain nematic elastomers [5]. Quantitative, but not qualitative, deviations from characteristically soft deformations occur if the elastomer is only semisoft, that is if it deviates from ideal symmetry requirements [6]. We shall find this general idea applicable also in certain types of smectic elastomers.

Monodomain smectic A (SmA) elastomers, synthesized by loading a swollen sample in the isotropic state and deswelling it into the smectic state, do not show any sign of soft elasticity. The smectic $\mathrm{C}(\mathrm{SmC})$ phase has also been synthesized experimentally and been formed into monodomains [7]. This phase is significant technologically because of its ferroelectric properties [8,9]. Typically during soft deformation, shape changes accompany director rotation. Once the director is anchored perpendicular to the layers and loses its freedom to move relative to the solid matrix, softness is lost. In the SmC phase the director has sufficient additional freedom to be soft because the constraint on the layer spacing does not totally determine the orientation of the polymer shape tensor. Biaxial SmA is a conceptually simpler phase and also has sufficient freedom, but is of more limited experimental interest. Models of these two phases have been studied theoretically by different methods of nonlinear elasticity from ours, but only for small strains, and shown to be soft [10]. We aim here to give explicit geometrical forms of soft modes by calculating the required deformation tensor for monodomains of these two phases of smectic elastomers. A simple illustration is first given for biaxial SmA, but our main aim is SmC elastomers. In Sec. II we summarize the model that will be used. We then calculate the soft modes of biaxial SmA elastomers in Sec. III. Finally we calculate the soft modes of an SmC elastomer in Sec. IV. The reader not interested in the SmA illustration can proceed directly from Sec. II to Sec. IV. Explicit forms of the SmC soft modes are given in Sec. IV. We delay addressing the related, important question of the spontaneous shears that develop during the symmetry breaking transition $\mathrm{SmA} \rightarrow \mathrm{SmC}$. In another paper we relate such shears to the amplitude of the soft modes discussed here. Our main conclusion is that as a result of the layer spacing constraint, the soft modes of a smectic elastomer are defined by only one parameter (excluding an arbitrary body rotation). This can be chosen as the azimuthal rotation of the director around the layer normal.

\section{SMECTIC ELASTOMER MODEL}

The models of biaxial SmA and SmC elastomers we will use here are extensions of the SmA elastomer model derived in [11] which we now summarize. The end-to-end span of the polymer chains that make up the elastomer are modeled by an anisotropic Gaussian distribution, as in nematic elastomers, which obtains when chains are sufficiently long and which ensures that the matrix formed on cross-linking is rub-
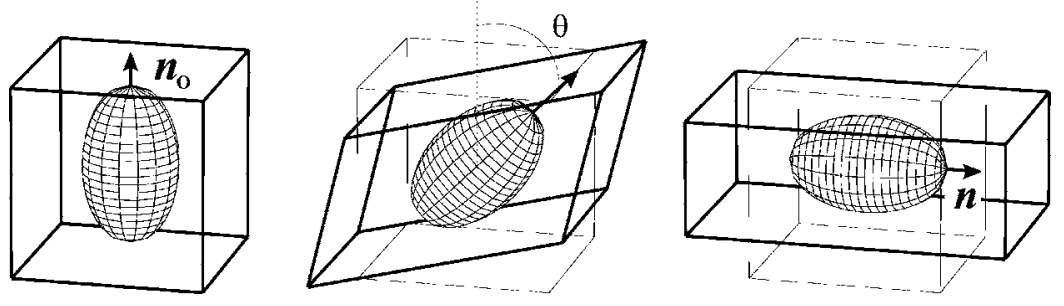

011703-1
FIG. 1. Chain shape distribution is rotated by $90^{\circ}$ from its initial direction $\mathbf{n}_{0}$ to a perpendicular n with an intermediate state direction at $\theta$ shown. The rubber deforms to accommodate the changing chain shape distribution without distorting it. 
bery and highly extensible. Additionally, in smectics the effect of the layers is to create a periodic potential which biases where the cross-link points, and thus the ends of the network strands, sit. As a result of this potential the crosslink point distribution in the elastomer is only homogeneous within the plane of the layers. Parallel to the layer normal the distribution is periodic, as cross-link points sink into potential wells. This picture of smectic elastomers results in a strong coupling of the matrix to the layers. Consequently, SmA elastomers have a modulus comparable to that penalizing the change of smectic layer spacing for any shape changes of the rubber matrix which convect layers to new spacings. This is a dramatic effect - smectic elastomers are rubbery in two dimensions and solidlike in the third; at larger strains they then suffer rotational instabilities. This reduces the cost of distortions involving layer spacing change to those involving the lower cost of rubbery distortion. In SmA elastomers there is close agreement between experiment [12] and theory [11].

We shall need the tensor $\ell_{0}$ of effective step lengths of the polymer at the instant of cross-linking. It is defined by the second moment that specifies entirely the Gaussian distribution of chains at that time:

$$
\left\langle R_{\alpha}^{0} R_{\beta}^{0}\right\rangle p_{0}=\frac{1}{3} L \ell_{0 \alpha \beta} .
$$

In the uniaxial case in the principal frame there are two step lengths, $\ell_{\|}$parallel to the (unit) director $\mathbf{n}$ specifying the direction of order, and $\ell_{\perp}$ perpendicular to $\mathbf{n}$. Note that on undergoing a phase change from the nematic to a smectic state, the degree of anisotropy of the polymer, $r=\ell_{\|} / \ell_{\perp}$, may be changed because of the additional penalty the polymer chains pay for crossing the smectic layers. In the SmA phase this effect can be described by decreasing the value of $r$ for prolate chains on transition to the smectic phase. This effect in SmC elastomers is more complicated, as will be discussed in Sec. IV.

We follow similar microscopic models of nematic elastomers in assuming that the smectic elastomer is composed of cross-linked polymers with identical strand length $L$, and that the cross-link points deform affinely, that is according to

$$
\mathbf{R}=\underline{\underline{\lambda}} \cdot \mathbf{R}_{0} .
$$

This is approximately true for individual chains. It is correct on average and is exact in the limit of infinite cross-link point functionality. The deviation from Eq. (2) for finite functionalities is small - a change in the prefactor of the free energy. The quenched average of the elastic free energy density over the end positions, $\mathbf{R}_{1,2}$, and over the layers that the cross-link points occupy (assuming a strong potential localizing cross-links to layers) can be performed as in [11]. The resulting free energy density has a term proportional to $\mathcal{V}^{2 / 3}$ (where $\mathcal{V}$ is the volume of the system) penalizing translations of the layers relative to the rubber matrix. This divergent intensive term enforces the affine deformation of the smectic layers with the matrix (crosslink points):

$$
\mathbf{q}=\underline{\underline{\lambda}}^{-T} \cdot \mathbf{q}_{0}
$$

where $\mathbf{q}_{0}$ is the initial wave vector, and $\mathbf{q}$ the current wave vector of the smectic layers, and $\underline{\underline{\lambda}}^{-T}$ denotes the inverse transpose of the deformation matrix. The affine deformation of layers Eq. (3) makes the layer spacing

$$
\frac{d}{d_{0}}=\frac{1}{\left|\underline{\underline{\lambda}}^{-T} \cdot \mathbf{k}_{0}\right|},
$$

where $\mathbf{k}_{0}$ is the initial (unit) direction of the layer normal in the solid, $\mathbf{k}_{0}=\mathbf{q}_{0} / q_{0}, d$ is the current layer spacing, and $d_{0}$ is the initial layer spacing. The wave vector magnitude is $q_{0}$ $=2 \pi / d_{0}$.

If the rubber-elastic free energy terms and the smectic layer modulus terms are collected together then the resulting free energy density expression is

$$
f=\frac{1}{2} \mu \operatorname{Tr}\left[\underline{\underline{\lambda}} \cdot \underline{\ell}_{0} \cdot \underline{\underline{\lambda}}^{T} \cdot \underline{\underline{\ell}}_{\mathbf{n}}^{-1}\right]+\frac{1}{2} B\left(\frac{d}{d_{0}}-1\right)^{2},
$$

where $B$ is the smectic modulus associated with deforming the layer spacing.

Both terms in the free energy density are non-negative. If we are to find deformations of zero cost, then the second term must vanish, that is $d=d_{0}$ during deformations. This condition is easily expressed from Eq. (4) [or from Eq. (3) with $\left.|\mathbf{q}|=q_{0}\right]$, that is one must take $\underline{\underline{\lambda}}$ obeying the constraint:

$$
\mathbf{k}_{0}^{T} \cdot \underline{\underline{\lambda}}^{-1} \cdot \underline{\underline{\lambda}}^{-T} \cdot \mathbf{k}_{0}=1
$$

The first, rubber-elastic term of Eq. (5) is identical to that of purely nematic elastomers and arises from distorting the chain distribution away from its optimal shape. The minimum energy density is $3 \mu / 2$ which arises when the $\underline{\underline{\lambda}}$ is such that the argument of the trace reduces to the unit tensor $\underline{\underline{\delta}}$. Trivially this can be satisfied by no deformation and no director rotation, or by a director rotation and a body rotation that are the same. The general form of nontrivial, soft modes in nematic elastomers [3] is

$$
\underline{\underline{\lambda}}=\underline{\underline{\ell}}_{\mathbf{n}}^{1 / 2} \cdot \underline{\underline{W}} \cdot \underline{\underline{\ell}}_{0}^{-1 / 2},
$$

where $\underline{\underline{W}}$ is a general rotation matrix, $\ell_{0}$ is the initial anisotropy tensor, and $\underline{\underline{\ell}}_{\mathbf{n}}$ is the current anisotropy tensor of the polymer chain distribution, dependent on director $\mathbf{n}$. One can readily check that the $\underline{\underline{\lambda}}$ of Eq. (7) renders the argument of the trace in Eq. (5) equal to $\underline{\underline{\delta}}$ and hence gives the minimal free energy.

The tensor $\ell_{\mathbf{n}}=\left(\ell_{\|}-\ell_{\perp}\right) \mathbf{n n}^{T}+\underline{\underline{\delta}} \rightarrow \ell_{\perp}\left[(r-1) \mathbf{n n}^{T}+\underline{\underline{\delta}}\right]$ can have a factor of $\ell_{\perp}$ extracted which we henceforth ignore since $\ell$ always appears in expressions with an $\ell^{-1}$, and thus with an associated, cancelling $1 / \ell_{\perp}$ factor, if the latter tensor is also taken in its reduced form. In such forms $\underline{\underline{n}}_{\mathbf{n}}^{1 / 2}=(\sqrt{r}$ $-1) \mathbf{n n}^{T}+\underline{\underline{\delta}}$ and $\ell_{\underline{\mathbf{n}}}^{-1 / 2}=(1 / \sqrt{r}-1) \mathbf{n n}^{T}+\underline{\underline{\delta}}$. Expressions ${ }^{\prime}$ we later require, such as $\ell_{\mathbf{n}}^{1 / 2} \cdot \mathbf{n} \rightarrow \sqrt{r} \mathbf{n}$, are then easy to evaluate.

The general form Eq. (7) can be rewritten in terms of a particular vector $\mathbf{n}^{\prime}$ such that $\mathbf{n}=\underline{\underline{W}} \cdot \mathbf{n}^{\prime}$ by multiplying it by $\underline{\underline{W}} \cdot \underline{\underline{W}}^{T}$ from the left. The factor $\underline{\underline{W}}^{T} \cdot \underline{\underline{n}}_{\mathbf{n}}^{1 / 2} \cdot \underline{\underline{W}}$ becomes $\underline{\underline{\ell}}_{\mathbf{n}^{\prime}}^{1 / 2}$ since the effect of the $\underline{\underline{W}}$ tensors is a rotation of the tensor $\ell_{\mathbf{n}}^{1 / 2}$. Thus the soft mode (7) becomes 


$$
\underline{\underline{\lambda}}=\underline{\underline{W}} \cdot{\underline{\underline{\mathbf{n}^{\prime}}}}^{1 / 2} \cdot \underline{\underline{\ell}}_{0}^{-1 / 2} .
$$

In rewriting the deformation in this way it is clear that the shape of the body after a soft deformation is determined by the choice of $\mathbf{n}^{\prime}$ and is given by the term $\ell_{=}^{1 / 2} \cdot \ell_{0}^{-1 / 2}$ (which may contain an element of body rotation which we shall extract). It is followed by $\underline{\underline{W}}$ which is a pure body rotation and is of no elastic significance. We can choose the vector $\mathbf{n}^{\prime}$ anywhere on the surface of a unit sphere. Consequently a nematic elastomer has several ways in which it can respond softly to an imposed deformation. In the case of a smectic elastomer, however, there is considerably less freedom because of the layer spacing constraint.

\section{BIAXIAL SMECTIC A ELASTOMERS}

Biaxial nematic phases, that is liquid crystalline phases where there is ordering along the director and perpendicular to it, are rare. Examples of both lyotropic and thermotropic biaxial nematic liquid crystals are known [13,14]. Biaxial nematic phases have also been found in nematic polymers $[15,16]$ and in principle it should be possible to make a biaxial nematic liquid crystal elastomer. To our knowledge these have not yet been made, but as in [10] we calculate their soft elastic deformations as an illustration since they are so much simpler than $\mathrm{SmC}$ elastomers.

To model biaxial SmA elastomers we follow Sec. II but include a biaxial polymer shape tensor, $\ell$, with principal axes $\mathbf{n}, \mathbf{m}$, and $\mathbf{l}$, and corresponding eigenvalues $\ell_{\|}, \ell_{1}$, and $\ell_{2}$. The shape tensor of a biaxial polymer is thus

$$
\underline{\ell}=\left(\begin{array}{ccc}
\ell_{1} & 0 & 0 \\
0 & \ell_{2} & 0 \\
0 & 0 & \ell_{\|}
\end{array}\right)
$$

In the soft mode of Fig. 1, director rotation was about an axis perpendicular to $\mathbf{n}_{0}$. In SmA elastomers the layer normal $\mathbf{k}$ and the director $\mathbf{n}$ (the primary alignment direction) are identified and such a soft mode is now lost, as we prove below. However in a biaxial SmA, rotation about $\mathbf{n}$ is now nontrivial. The secondary alignment axes are free to rotate in the plane of the layers. Since this section of $\ell$ is noncircular, $\ell_{1} \neq \ell_{2}$, soft deformations arise in complete änalogy to Fig. 1 for nematics but in the plane perpendicular to $\mathbf{n}_{0}$, as we also prove below.

The layer spacing constraint Eq. (6) on identifying $\mathbf{k}$ and n becomes

$$
\mathbf{n}_{0}^{T} \cdot \underline{\underline{\lambda}}^{-1} \cdot \underline{\underline{\lambda}}^{-T} \cdot \mathbf{n}_{0}=1
$$

The general soft mode (7) in constraint (10) yields

$$
\frac{1}{r}=\mathbf{n}_{0}^{T} \cdot \underline{\underline{W^{T}}} \cdot \underline{\underline{\ell_{\mathbf{n}}^{-1}}} \cdot \underline{\underline{W}} \cdot \mathbf{n}_{0} .
$$

Deriving Eq. (11) from Eq. (10) requires expressions discussed after Eq. (7). The only solution to this equation is $\mathbf{n}$ $=\mathbf{n}^{\prime} \equiv \underline{\underline{W}} \cdot \mathbf{n}_{0}$ because the quadric surface associated with $\ell^{-1}$ only has the correct width at one point (the maximum or minimum width depending on whether $r<1$ or $r>1$ ). Re- turning this $\underline{\underline{W}}$ to Eq. (7) gives, on using the forms for $\ell^{1 / 2}$ and $\ell_{0}^{-1 / 2}$, the result $\underline{\underline{\lambda}}=\underline{\underline{W}}$, that is a trivial body rotation $=$ the same amount as the director has been rotated. There has been no shape change of the body. Thus there is indeed no freedom for the primary alignment director to rotate with respect to the matrix, and hence no soft modes in uniaxial SmA elastomers except for pure rotations. This is because if the layer normal $\mathbf{n}$ is moved by any deformation other than a rotation then the layer spacing will be changed and the resulting state will be higher in energy. However, in biaxial SmA elastomers the secondary alignment axes are unconstrained, thus there is still enough freedom for a soft mode to exist, that is a manifold of solutions of Eq. (11) not involving rotation of $\mathbf{n}$.

The soft modes can be decomposed into their component rotations to gain a better understanding of them as follows. First we decompose the general $\underline{\underline{W}}$ matrix as

$$
\underline{\underline{W}}=\underline{\underline{W}} \mathbf{R} \cdot \underline{\underline{W}}_{\mathbf{n}_{0}}
$$

where $\underline{\underline{W}}_{\mathbf{n}_{0}}$ is a rotation about $\mathbf{n}_{0}$ and accordingly only reorients $\mathbf{m}_{0}$ and $\mathbf{l}_{0}$ in the plane perpendicular to $\mathbf{n}_{0}$. The choice of $\underline{\underline{W}} \underline{\mathbf{R}}_{\mathbf{W}}$ is somewhat special - it takes $\mathbf{n}_{0}$ to $\mathbf{n}$, that is $\mathbf{n}$ $=\underline{\underline{W}}_{\mathbf{R}} \cdot \mathbf{n}_{0}$ and thus $\mathbf{n}^{\prime}=\mathbf{n}_{0}$. Using this in Eq. (7) results in the following expression for the soft mode:

$$
\begin{aligned}
\underline{\underline{\lambda}} & =\underline{\ell}^{1 / 2} \cdot \underline{\underline{W}} \mathbf{R} \cdot \underline{\underline{W}}_{\mathbf{n}_{0}} \cdot \ell_{0}^{-1 / 2} \\
& =\underline{\underline{W}}_{\mathbf{R}} \cdot \underline{\underline{W}}{\underline{\mathbf{n}_{0}}}_{0} \cdot\left[\underline{\underline{\ell}}_{\mathbf{n}_{0}, \mathbf{m}^{\prime}, \mathbf{l}^{\prime}} \cdot \ell_{0}^{-1 / 2}\right],
\end{aligned}
$$

the second form arising from the same arguments that led to Eq. (8) from Eq. (7). The factor in square brackets here is a soft mode in which the secondary alignment axes rotate around the fixed primary axis. It clearly leaves the layer structure unchanged (the $\mathbf{n}_{0}$ orientation in $\ell^{1 / 2}$ ) and explains the choice of $\underline{\underline{W}}_{\mathbf{R}}$. The vector $\mathbf{m}^{\prime}$ is defined $\overline{\bar{b}} \mathrm{y} \mathbf{m}=\underline{\underline{W}} \cdot \mathbf{m}^{\prime}$ and likewise for $\mathbf{l}^{\prime}$. This part of the deformation is given by

$$
\left(\begin{array}{ccc}
1-\left(1-1 / \sqrt{r_{\perp}}\right) \sin ^{2} \phi & \left(1-\sqrt{r_{\perp}}\right) \sin \phi \cos \phi & 0 \\
\left(1 / \sqrt{r_{\perp}}-1\right) \sin \phi \cos \phi & 1+\left(\sqrt{r_{\perp}}-1\right) \sin ^{2} \phi & 0 \\
0 & 0 & 1
\end{array}\right),
$$

where $\phi$ is the angle that the secondary alignment axes have rotated through and $r_{\perp}=\ell_{1} / \ell_{2}$ is a material parameter expressing the degree of biaxiality in the chain anisotropy. The $\underline{\underline{W}} \mathbf{R}_{\mathbf{R}} \cdot \underline{\underline{W}}_{\mathbf{n}_{0}}$ that follows this deformation is a trivial body rotation which we can neglect. Thus all soft modes in biaxial SmA elastomers can be decomposed into a body rotation that follows the soft mode that has the primary alignment axis fixed and the secondary alignment axes rotated from their initial orientation.

\section{Example: Imposed $\boldsymbol{\lambda}_{y y}$}

For later comparison with the soft modes of a SmC elastomer, we present the soft mode associated with an imposed $\lambda_{y y}$. This mode has a fixed primary alignment direction, $\mathbf{n}$ $=\mathbf{n}_{0}$, but mobile secondary alignment directions, $\mathbf{m}$ and $\mathbf{l}$; see Fig. 2. The center of the diagram depicts the secondary alignment axis $\mathbf{m}$ which is initially along the $\mathbf{x}$ direction and is 


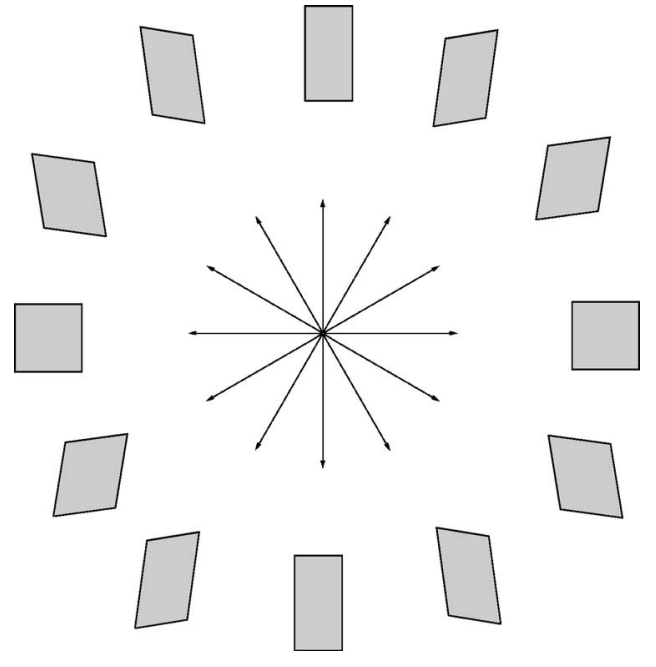

FIG. 2. The soft mode of a biaxial SmA elastomer of anisotropy $r_{\perp}=2$, with the layer normal out of the page. The direction of the secondary director $\mathbf{m}$ is shown in the center. It rotates by $\phi=30^{\circ}$ from one image to the next.

associated with $\ell_{1}\left(>\ell_{2}\right.$ and thus $\left.r_{\perp}>1\right)$. We are therefore extending along the initially shorter perpendicular direction. Along the outside of the diagram the shape of the biaxial SmA elastomer is viewed from along the layer normal. Note that the maximum extension in the $\mathbf{y}$ direction is by a factor of $\sqrt{r_{\perp}}$ and occurs when these secondary alignment axes have rotated by $\phi=90^{\circ}$.

\section{SMECTIC C ELASTOMERS}

For SmC elastomers we extend the SmA elastomer model presented in Sec. II. Now the director is tilted with respect to the layer normal. We denote the layer normal by $\mathbf{k}$, the director by $\mathbf{n}$, and the direction in which the mesogens are tilted in by c, all unit vectors (see Fig. 3). Thus if the director has a tilt angle of $\theta$ then

$$
\mathbf{n}=\mathbf{k} \cos \theta+\mathbf{c} \sin \theta .
$$

The constraint (6) on the layer spacing (4) must still be obeyed, but this does not constrain the whole of the director now, giving scope for soft modes.

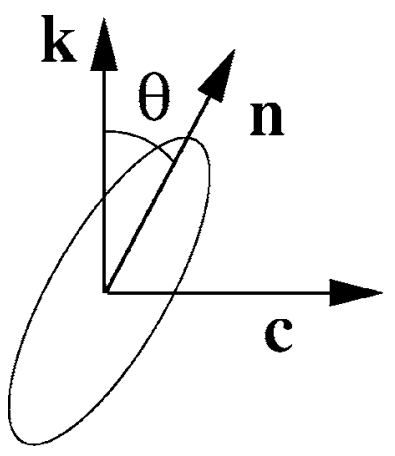

FIG. 3. In a SmC elastomer the director, $\mathbf{n}$, is tilted with respect to the layer normal, $\mathbf{k}$, by an angle $\theta$.
Here we use a uniaxial tensor to represent the polymer shape anisotropy for simplicity. However, in the SmC phase there are two distinguished directions, the layer normal and the director, which should alter the polymer shape anisotropy. Thus it is anticipated that the shape tensor should really be biaxial. However, we shall find that soft elasticity already arises because of the biaxial arrangement of a uniaxial $\ell$ tilted at a fixed angle to the layer normal. The same qualitative behavior is expected for a truly biaxial $\ell$ as that generated below by the simplification we follow.

Another term in this model penalizing the tilt of the director away from making an angle $\theta$ with the layer normal could be included. However, since the main concern here is soft modes, deviation from tilt $\theta$ with any energy cost would remove softness during deformation. Consequently, this term is not included here and, as with the layer spacing, the tilt angle is simply regarded as being fixed. In any event, away from the $\mathrm{SmA} \rightarrow \mathrm{SmC}$ transition it is expected that the modulus associated with changes in $\theta$ is very high.

\section{A. General form of soft modes}

Our model of SmC elastomers has underpinning nematic rubber elasticity with constraints of constancy of layer spacing. We again start with general soft modes of nematic elastomers Eq. (7), with the constraint on $\underline{\lambda}$ Eq. (6). We introduce a new auxiliary vector, $\mathbf{w}_{0}=\ell_{0}^{1 \overline{\overline{1}}} \cdot \mathbf{k}_{0}$, much as we introduced the auxiliary $\mathbf{n}^{\prime}$ to the director $\mathbf{n}$. With the help of $\underline{\ell}_{0}^{1 / 2}=(\sqrt{r}-1) \mathbf{n}_{0} \mathbf{n}_{0}^{T}+\underline{\underline{\delta}}$ one has

$$
\mathbf{w}_{0}=\underline{\ell_{0}^{1 / 2}} \cdot \mathbf{k}_{0}=\mathbf{k}_{0}+(\sqrt{r}-1) \cos \theta \mathbf{n}_{0} .
$$

Thus $\mathbf{n}_{0}, \mathbf{k}_{0}$, and $\mathbf{w}_{0}$ lie in the same plane, with $\mathbf{w}_{0}$ between $\mathbf{n}_{0}$ and $\mathbf{k}_{0}$ for $r>1$. The modulus of $\mathbf{w}_{0}$ is not unity but rather $w_{0}^{2}=1+(r-1) \cos ^{2} \theta>1$ for $r>1$.

Inserting the general soft mode (7) into the layer spacing constraint, Eq. (6), and using $\mathbf{w}_{0}$, one obtains

$$
\mathbf{w}_{0}^{T} \cdot \underline{\underline{W}}^{T} \cdot \underline{\underline{\ell}}_{\mathbf{n}}^{-1} \cdot \underline{\underline{W}} \cdot \mathbf{w}_{0}=1 .
$$

The tensor $\underline{\ell}_{\mathbf{n}}^{-1}=\left(\frac{1}{r}-1\right) \mathbf{n n}^{T}+\underline{\underline{\delta}}$ can be injected into Eq. (17), whence $\quad 1 \stackrel{=}{=}\left(\frac{1}{r}-1\right)\left[\mathbf{n}^{T} \cdot \underline{\underline{W}} \cdot \mathbf{w}_{0}\right]^{2}+\mathbf{w}_{0}^{T} \cdot \mathbf{w}_{0}$. Using $w_{0}^{2}=1+(r$ $-1) \cos ^{2} \theta$, this constraint can be rearranged into the more transparent form:

$$
\left(\underline{\underline{W}} \cdot \mathbf{w}_{0}\right)^{T} \cdot \mathbf{n}= \pm \sqrt{r} \cos \theta
$$

That is, Eq. (18) is the equation of two planes that the tip of the director $\mathbf{n}$ sits on. They are a distance: $\pm 1 /(1$ $\left.+\frac{1}{r} \tan ^{2} \theta\right)^{1 / 2}$ from the origin, and with normal vector along the $\underline{\underline{W}} \cdot \mathbf{w}_{0}$ direction. Additionally the director is a unit vector and thus the allowed directors $\mathbf{n}$ after distortion sit on the intersection of the planes with the unit sphere $\mathbf{n} \cdot \mathbf{n}=1$. These circles of intersection are always guaranteed since the distance of the planes from the origin is less than unity.

The constraint of constant layer spacing having been implemented, we now need to ensure that the direction of the layer normal changes affinely with the matrix, Eq. (3). The new layer normal follows from 


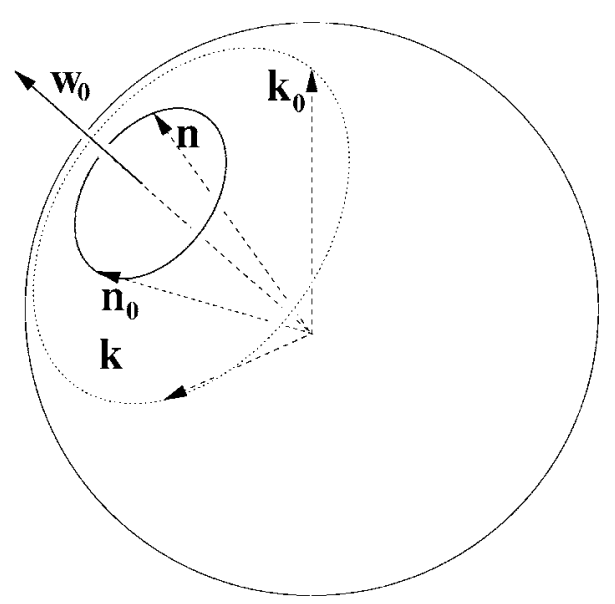

FIG. 4. An illustration of the director orientations that satisfy the layer constraint.

$$
\mathbf{k}=\frac{\underline{\underline{\lambda}}^{-T} \cdot \mathbf{k}_{0}}{\left|\underline{\underline{\lambda}}^{-T} \cdot \mathbf{k}_{0}\right|} \rightarrow \underline{\underline{\lambda}}^{-T} \cdot \mathbf{k}_{0},
$$

which is a normalized form of the transformed layer wave vector, Eq. (3), and simplifies as shown if the layer spacing is fixed, that is $d / d_{0}=1$ in Eq. (4) which means equally that Eq. (6) is satisfied. When considering a particular soft deformation constructed by choosing a particular $W$ in Eq. (7), the above determines the choice of $\underline{\underline{W}}$ if we want to achieve a desired $\mathbf{n}$ and $\mathbf{k}$. We insert a soft $\underline{\bar{\lambda}}^{-T}$ into this expression for the new k. It is $\underline{\underline{\lambda}}^{-T}=\underline{\ell}_{\mathbf{n}}^{-1 / 2} \cdot \underline{\underline{W}} \cdot \underline{\ell}_{0}^{1 / 2}$, on inverting and transposing Eq. (7), whereupon the normal becomes $\mathbf{k}=\underline{\ell}_{\mathbf{n}}^{-1 / 2} \cdot \underline{\underline{W}} \cdot \mathbf{w}_{0}$. Multiplying both sides from the left by $\ell_{\mathbf{n}}^{1 / 2}$, using the explicit form for $\ell_{\mathbf{n}}^{1 / 2}$, and recalling $\mathbf{n} \cdot \mathbf{k}=\cos \theta$, one obtains

$$
\underline{\underline{W}} \cdot \mathbf{w}_{0}=\mathbf{k}+(\sqrt{r}-1) \cos \theta \mathbf{n} .
$$

Thus the three vectors $\underline{\underline{W}} \cdot \mathbf{w}_{0}, \mathbf{k}$, and $\mathbf{n}$ obey the relation of the same form as Eq. (16). This motivates the definition of a new auxiliary vector $\underline{\underline{W}} \cdot \mathbf{w}_{0}=\mathbf{w}$.

The allowed soft modes correspond to the points where the two planes defined in Eq. (18) intersect a sphere. To analyze the soft modes first the case without $\underline{\underline{W}}$ is studied, followed by the more general case of those modes including a matrix $\underline{\underline{W}}$.

\section{Geometrical interpretation of soft modes of the form $\underline{\ell_{\mathrm{n}}^{1 / 2} \cdot \ell_{0}^{-1 / 2}}$}

The algebraically simplest (but certainly not physically simplest) soft modes in an $\mathrm{SmC}$ elastomer are those without a $\underline{\underline{W}}$ matrix. In this case the final director must lie on the intersection of the unit sphere and the planes of Eq. (18). An illustration of this is shown in Fig. 4. The figure shows the initial director $\mathbf{n}_{0}$ and the initial layer normal $\mathbf{k}_{0}$ and two circles on the surface of the sphere, corresponding to the final orientation of the director $\mathbf{n}$ and layer normal $\mathbf{k}$, that obey the layer constraint. Note that not all final positions of the director are possible with soft modes of this form. For $\mathbf{n}$ not on the circle of Fig. 4, an appropriate $\underline{\underline{W}}$ must be included such that the required final director position still obeys the layer constraint.

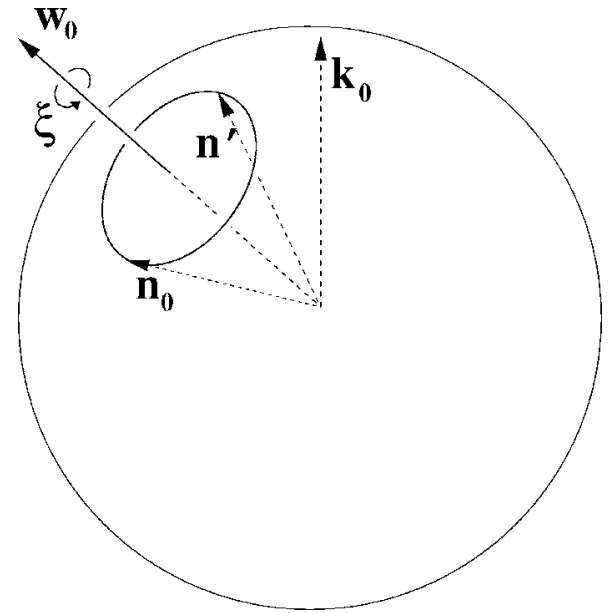

FIG. 5. The first stage of the calculation of a general soft mode: rotating the initial director $\mathbf{n}_{0}$ around to $\mathbf{n}^{\prime}$.

\section{Geometrical interpretation of general soft modes of the form $\underline{\ell_{\mathrm{n}}^{1 / 2}} \cdot \underline{\underline{W}} \cdot \stackrel{\ell_{0}^{-1 / 2}}{=}$}

As in Eq. (8) we reexpress the general soft mode as $\underline{\underline{\lambda}}$ $=\underline{\underline{W}} \cdot \underline{\ell}_{\mathbf{n}^{\prime}}^{1 / 2} \cdot \underline{\ell}_{0}^{-1 / 2}$ with a fictitious director $\mathbf{n}^{\prime}$, defined by $\underline{\underline{W}}^{T} \cdot \mathbf{n}=\mathbf{n}^{\prime}$, playing the role of $\mathbf{n}$ in the simple soft mode of Fig. 4, see now Fig. 5. We break $\underline{\underline{W}}$ down into two successive rotations, $\underline{\underline{W}}=\underline{\underline{\underline{W}}} \underline{\mathbf{R}}_{\underline{\underline{W}}} \underline{\mathbf{w}}_{0}(\xi)$ where the latter is a rotation by $\xi$ about $\mathbf{w}_{0}$ and takes $\mathbf{n}^{\prime}$ to $\mathbf{n}_{0}$, and $\underline{\underline{W}}_{\mathbf{R}}$ takes $\mathbf{n}_{0}$ to $\mathbf{n}$. Specifically $\underline{\underline{W}}_{\mathbf{R}}^{T} \cdot \mathbf{n}=\mathbf{n}_{0}$ and $\underline{\underline{W}}_{\mathbf{w}_{0}}^{T} \cdot \mathbf{n}=\mathbf{n}^{\prime}$ and one explicitly sees $\underline{\underline{\ell}}_{\mathbf{n}^{\prime}}^{1 / 2}$ $=\underline{\underline{W}}_{\mathbf{w}_{0}}^{T} \cdot \underline{\underline{\underline{W}}} \underline{\mathbf{R}}^{T} \cdot \underline{\ell}_{\mathbf{n}}^{1 / 2} \cdot \underline{\underline{\underline{W}}} \mathbf{R} \cdot \underline{\underline{\underline{W}}}_{\mathbf{w}_{0}} \cdot$ The power of the method is that $\underline{\underline{\lambda}}$ is now

$$
\underline{\underline{\lambda}}=\underline{\underline{W}} \mathbf{R} \cdot \underline{\underline{W}}_{\mathbf{w}_{0}}(\xi) \cdot{\underline{\underline{\ell^{\prime}}}}_{\mathbf{n}^{\prime}}^{1 / 2} \cdot \underline{\ell}_{0}^{1 / 2}
$$

where the soft mode ${\underline{\mathbf{n}^{\prime}}}_{\mathbf{n}^{\prime}}^{-1 / 2} \cdot \ell_{0}^{1 / 2}$ is parametrized by the angle $\xi$ and is independent of $\underline{\underline{\underline{W}}} \mathbf{R}$ which we can freely set in order to obtain whatever final director we desire, that is $\mathbf{n}=\underline{\underline{R}} \cdot \mathbf{n}_{0}$. This general, final director is not confined to the circle about

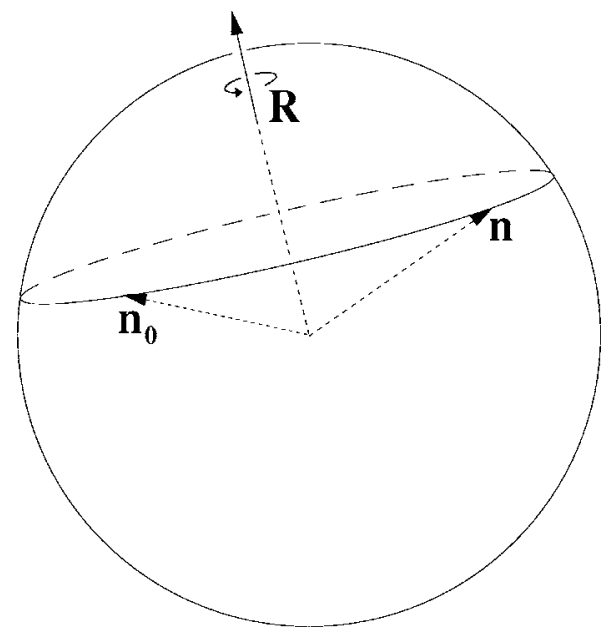

FIG. 6. The second stage of the calculation of a soft mode: a general rotation about the axis $\mathbf{R}$. 


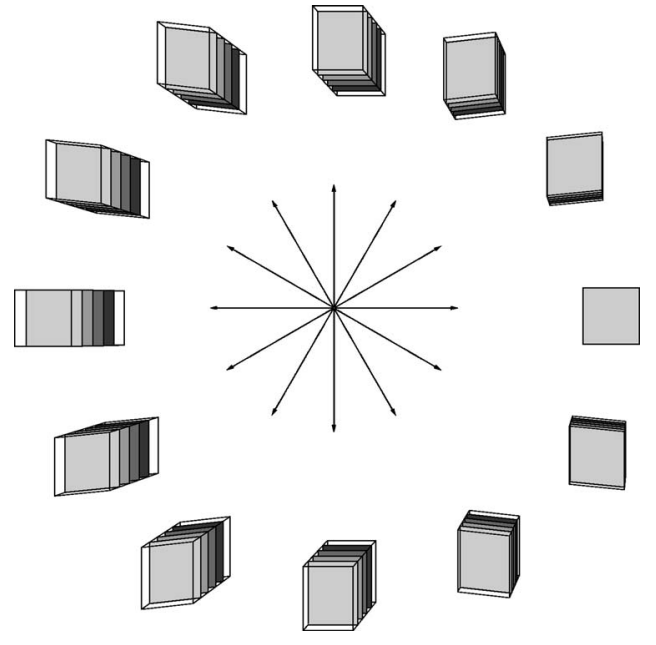

FIG. 7. An illustration of the soft mode of a SmC elastomer. In this case the layer normal remains out of the page and the $\mathbf{c}$ direction together with $\phi$ is shown in the center of the diagram. A tilt angle of $\theta=30^{\circ}$ and an anisotropy of $r=8$ were chosen.

$\mathbf{w}_{0}$, see Fig. 6. Having decided where the final director is to point, one then applies the body rotation $\underline{\underline{W}}_{\mathbf{R}} \cdot \underline{\underline{W}}_{\mathbf{w}_{0}}(\xi)$ to the softly deformed sample to complete the deformation (21).

The element of body rotation of the underlying soft mode can be separated out, leaving just a symmetric deformation, by using the polar decomposition theorem:

$$
\underline{\underline{\lambda}}=\underline{\underline{\ell}_{\mathbf{n}^{\prime}}^{1 / 2}} \cdot \underline{\underline{\ell}}_{0}^{-1 / 2}=\underline{\underline{U}} \cdot \underline{\underline{S}},
$$

where $\underline{\underline{U}}$ is a rotation matrix and $\underline{\underline{S}}$ is a symmetric matrix. The rotation axis for this decomposition must be in the $\mathbf{n}^{\prime} \wedge \mathbf{n}_{0}$ direction, since soft modes can be thought of as being parametrically generated by the rotation of the director [4], in this case about the axis $\mathbf{n}^{\prime} \wedge \mathbf{n}_{0}$. We can use this information to construct $\underline{\underline{U}}^{T} \cdot \underline{\underline{\lambda}}$ and demand that it is symmetric to find $\underline{\underline{S}}$. The resulting rotation angle is given by

$$
\tan \alpha=\frac{(1-\sqrt{r})^{2}\left(\mathbf{n}_{0} \cdot \mathbf{n}^{\prime}\right) \sqrt{1-\left(\mathbf{n}_{0} \cdot \mathbf{n}^{\prime}\right)^{2}}}{(1+r)-\left(\mathbf{n}_{0} \cdot \mathbf{n}^{\prime}\right)^{2}(1-\sqrt{r})^{2}} .
$$

Now a particular example of a soft mode, relevant to experiment, is considered. Here rigid clamping constraints are not included so there is no formation of microstructure.

\section{B. Example: Imposed $\lambda_{y y}$}

To illustrate the soft modes we could choose to impose an elongation in any direction, provided the director has scope to rotate into that direction and thereby to extend the sample. This excludes stretches parallel to $\mathbf{n}_{0}$. An elongation perpendicular to the layer normal is particularly simple because it does not induce the layer normal to rotate.

An extension in the $\mathbf{y}$ direction is imposed on an elastomer with its layer normal in the $\mathbf{z}$ direction and the in-plane component of the director in the $\mathbf{x}$ direction, i.e., $\mathbf{c}=\mathbf{x}$ initially. We take a deformation matrix of the form

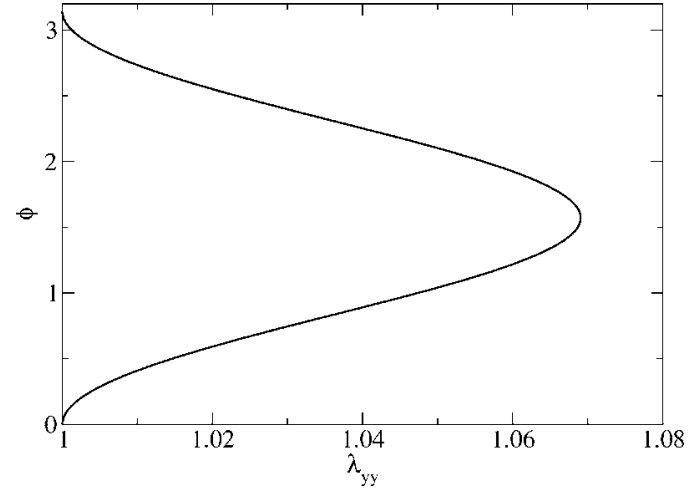

FIG. 8. Director rotation $\phi$ about the layer normal against elongation, $\lambda_{y y}$, perpendicular to its initial direction $\left(r=2, \theta=30^{\circ}\right)$.

$$
\underline{\underline{\lambda}}=\left(\begin{array}{ccc}
\lambda_{x x} & 0 & \lambda_{x z} \\
\lambda_{y x} & \lambda_{y y} & \lambda_{y z} \\
0 & 0 & \lambda_{z z}
\end{array}\right)
$$

where the components $\lambda_{x y}$ and $\lambda_{z y}$ are not included. This is because these $\lambda$ 's deform the sample by translating the $y$ faces of the sample in the $\pm \mathbf{x}$ and $\pm \mathbf{z}$ directions. Any small $y$ forces associated with the $y y$ elongation would generate counter torques and quickly eliminate $\lambda_{x y}$ and $\lambda_{z y}$. The $\lambda_{z x}$ component is excluded because without compensating elongation in the $\mathbf{z}$ direction it would compress the layers, see the analogous problem when a SmA is stretched along the layer normal [11]. It would also rotate a component of the director perpendicular to the stretch direction.

The initial orientation of the layer normal and the director are given by

$$
\mathbf{n}_{0}=(\sin \theta, 0, \cos \theta)
$$

$$
\mathbf{k}_{0}=(0,0,1)
$$

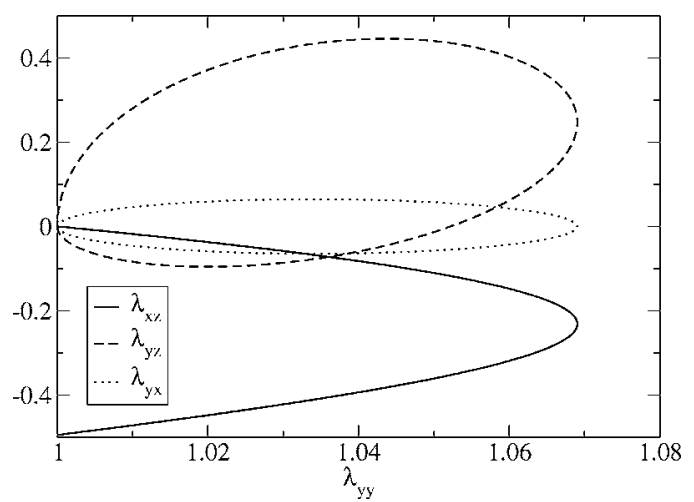

FIG. 9. Sympathetic shears $\lambda_{y x}, \lambda_{y z}$, and $\lambda_{x z}$ in response to an imposed $\lambda_{y y}$. Again $r=2$ and $\theta=30^{\circ}$. 
where $\theta$ is the tilt angle of the director (typically around $20^{\circ}$ ). The current orientation of the director is assumed to be

$$
\mathbf{n}=(\sin \theta \cos \phi, \sin \theta \sin \phi, \cos \theta),
$$

and the layer normal, $\mathbf{k}$ unmoved. The layer normal $\left(\mathbf{k}_{0}=\mathbf{z}\right)$ cannot be moved by deformation tensors of the form Eq. (24) since it must be derived from the expression $\mathbf{k}=\underline{\underline{\lambda}}^{-T} \cdot \mathbf{k}_{0}$ [i.e., the elements of $k_{i}$ are derived from the cofactors of the elements $\lambda_{i z}$, all of which vanish except for the cofactor of $\lambda_{z z}$, as can be seen by inspection of Eq. (24)]. In addition, the only consistent rotation matrices $\underline{\underline{W}}_{\mathbf{R}}$ that leave the layer normal unmoved must have their rotation axis, $\mathbf{R}$, parallel to $\mathbf{k}_{0}$. This rotation must take $\mathbf{n}_{0} \rightarrow \mathbf{n}$. Thus $\underline{\underline{W}}_{\mathbf{R}}$ can be identified as a rotation of angle $\phi$ around an axis parallel to $\mathbf{k}_{0}$, and could be written more concretely as $\underline{\underline{W}}_{\mathbf{k}_{0}}(\phi)$. Bearing this in mind, one constructs the tensor $\underline{\underline{\lambda}}=\underline{\underline{\underline{W}}} \mathbf{k}_{0}(\phi) \cdot \underline{\underline{W}} \mathbf{w}_{0}(\xi) \cdot \ell_{\mathbf{n}^{\prime}}^{-1 / 2} \cdot \ell_{0}^{-1 / 2}$ (for the details of this see the Appendix). The only remaining variable is $\xi$, and this can be determined by demanding that $\lambda_{x y}=0$ in the Appendix expression for $\underline{\underline{\lambda}}$. Writing $\rho=\sin ^{2} \theta$ $+r \cos ^{2} \theta \equiv w_{0}^{2}$, this yields the following equation for $\xi$ :

$$
0=\cos \xi \sin \phi+\sqrt{\frac{r}{\rho}} \cos \phi \sin \xi .
$$

Using this expression for $\xi$ all the components of the deformation tensor can be obtained. Defining $a(\phi)$ $=\sqrt{\cos ^{2} \phi+\rho / r \sin ^{2} \phi}$ we have the following matrix for $\underline{\underline{\lambda}}$ :

$$
\left(\begin{array}{ccc}
a(\phi) & 0 & \frac{(r-1) \sin 2 \theta}{2 \rho}[-a(\phi)+\cos \phi] \\
\left(1-\frac{\rho}{r}\right) \frac{\sin 2 \phi}{2 a(\phi)} & \frac{1}{a(\phi)} & \frac{(r-1) \sin 2 \theta}{2 \rho}\left[\sin \phi-\left(1-\frac{\rho}{r}\right) \frac{\sin 2 \phi}{2 a(\phi)}\right] \\
0 & 0 & 1
\end{array}\right)
$$

This tensor is explicitly constructed to be a soft mode and evidently has $\operatorname{det}[\underline{\underline{\lambda}}]=1$. To illustrate this mode Fig. 7 shows how this sample deforms for various different azimuthal angles, $\phi$. The figure gives a view of a block of SmC rubber down the layer normal and should be compared with Fig. 2. Note that even after a rotation of the director of $\phi=\pi$ the rubber does not return to its original configuration. Because of the tilt of the director with respect to the layer normal a strain $\lambda_{x z}<0$ is generated after $\phi \rightarrow \pi$ and this component has a $\cos \phi$ term. By contrast $\lambda_{y x}=\lambda_{y z}=0$ and $\lambda_{y y}=1$ at $\phi$ $=\pi$; indeed $\lambda_{y z}$ depends on $2 \phi$. At the intermediate value of $\phi=\pi / 2$ the elastomer has contracted along the direction of the original anisotropy tensor and so has developed both a $\lambda_{x z}$ and $\lambda_{y z}$ component of shear, that is with displacements in both the $\mathbf{x}$ and $\mathbf{y}$ directions. The maximum extension in the $\mathbf{y}$ direction occurs at $\phi=\pi / 2$, when the $\lambda_{y y}$ component takes the value $\sqrt{r / \rho}$. For the case with $\theta=30^{\circ}$ and $r=2$ this gives a maximum extension of roughly $7 \%$.

Alternatively, one can think of imposing the $\lambda_{y y}$ extension to induce the rotation $\phi$ of $\mathbf{c}$, Fig. 8, and the sympathetic shears $\lambda_{x z}, \lambda_{y z}$, and $\lambda_{y x}$, Fig. 9. These plots of the explicit forms given for the elements of $\underline{\underline{\lambda}}$ reveal singular edges to the rotation $\phi$ at $0, \pi$, and $2 \pi$ analogously to those seen in nematic soft elasticity. However for the example $r=2$ and $\theta=30^{\circ}$ the extent of soft extension is only $7 \%$.

It should be noted that the sample can develop very large shears softly; at $\phi=\pi, \lambda_{x z} \sim-\frac{1}{2}$, see Fig. 9. We address separately the details of the spontaneous mechanical shear of the elastomer in making the transition $\mathrm{SmA} \rightarrow \mathrm{SmC}$ which determines the amplitude of these soft modes.

\section{CONCLUSIONS}

We have presented a geometrical interpretation of the soft modes predicted in two phases of smectic elastomers. It was found that there is only one soft trajectory for the director, excluding body rotations, as a consequence of the restrictions imposed upon the elastomer by the fixed layer constraint. Specific examples of this mode were presented for monodomains of biaxial SmA or SmC. They can respond softly to a single imposed component of the deformation tensor. An illustration of imposed $\lambda_{y y}$ was given but in principle we could impose any other component, including $\lambda_{z z}$, through a combination of the single soft trajectory and a body rotation. The experimental boundary conditions of fixed ends near the clamps mean that any soft mode will be accompanied by microstructure, as is frequently the case in nematic elastomers.

\section{APPENDIX: DETAILS OF THE DEFORMATION MATRIX}

Here we give details of the calculation of the deformation tensor required for a soft mode when the $\lambda_{y y}$ component is imposed. The following vectors are required:

$$
\begin{gathered}
\mathbf{k}_{0}=(0,0,1), \\
\mathbf{c}_{0}=(0,1,0), \\
\mathbf{w}_{0}=\underline{\ell}_{0}^{1 / 2} \cdot \mathbf{k}_{0}, \\
\mathbf{c}=(\cos \phi, \sin \phi, 0),
\end{gathered}
$$




$$
\mathbf{k}=\mathbf{k}_{0}=\mathbf{R}
$$

From these vectors we can calculate the rotation matrices:

$$
\underline{\underline{W}}_{\mathbf{k}_{0}}(\phi)=\underline{\underline{\delta}} \cos \phi+(1-\cos \phi) \mathbf{k}_{0} \mathbf{k}_{0}^{T}+(\sin \phi) \mathbf{k}_{0} \wedge,
$$

$$
\underline{\underline{W}} \mathbf{w}_{0}(\xi)=\underline{\underline{\delta}} \cos \xi+\frac{(1-\cos \xi)}{w_{0}^{2}} \mathbf{w}_{0} \mathbf{w}_{0}^{T}+\frac{\sin \xi}{w_{0}} \mathbf{w}_{0} \wedge
$$

Using the expression

$$
\underline{\underline{\lambda}}=\underline{\underline{\ell_{\mathbf{n}}}} \underline{1 / 2} \cdot \underline{\underline{W}}_{\mathbf{k}_{0}}(\phi) \cdot \underline{\underline{W}}_{\mathbf{w}_{0}}(\xi) \cdot \underline{\underline{\ell}}_{0}^{-1 / 2}
$$

we obtain the following:

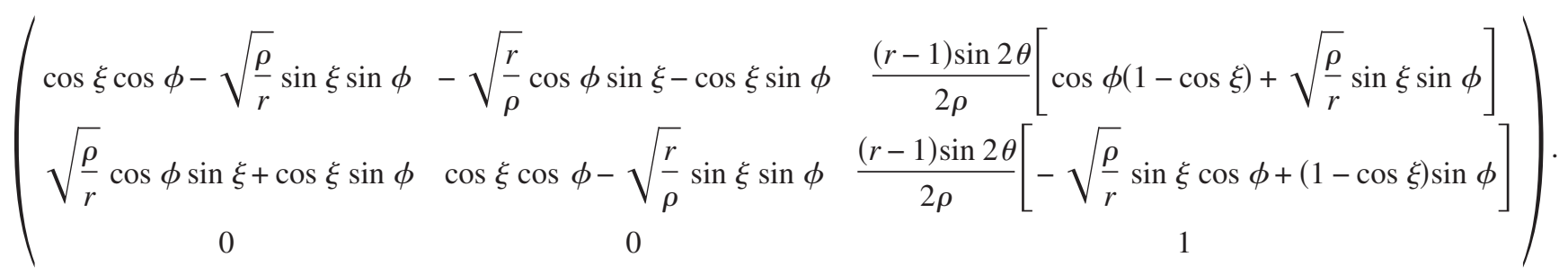

To determine $\xi$ we demand that the $\lambda_{x y}$ component is zero and obtain the matrix given in the text.

[1] L. Golubovic and T. C. Lubensky, Phys. Rev. Lett. 63, 1082 (1989).

[2] M. Warner, P. Bladon, and E. M. Terentjev, J. Phys. II 4, 93 (1994).

[3] P. D. Olmsted, J. Phys. II 4, 2215 (1994).

[4] M. Warner and E. Terentjev, Liquid Crystal Elastomers (Oxford University Press, Oxford, 2003).

[5] I. Kundler and H. Finkelmann, Macromol. Rapid Commun. 16, 679 (1995).

[6] H. Finkelmann, I. Kundler, E. Terentjev, and M. Warner, J. Phys. II 7, 1059 (1997).

[7] K. Hiraoka and H. Finkelmann, Macromol. Rapid Commun. 22, 456 (2001).

[8] W. Lehmann, H. Skupin, C. Tolksdorf, E. Gebhard, R. Zentel, P. Krüger, M. Lösche, and F. Kremer, Nature (London) 410, 447 (2001).
[9] K. Hiraoka, P. Stein, and H. Finkelmann, Macromol. Chem. Phys. 205, 48 (2004).

[10] O. Stenull and T. C. Lubensky, Phys. Rev. Lett. 94, 018304 (2005).

[11] J. M. Adams and M. Warner, Phys. Rev. E 71, 021708 (2005).

[12] E. Nishikawa and H. Finkelmann, Macromol. Chem. Phys. 200, 312 (1999).

[13] Y. Galerne and J. P. Marcerou, Phys. Rev. Lett. 51, 2109 (1983).

[14] B. R. Acharya, A. Primak, T. J. Dingermans, E. T. Samulski, and S. Kumar, Pramana, J. Phys. 61, 231 (2003).

[15] F. Hessel, R.-P. Herr, and H. Finkelmann, Macromol. Chem. Phys. 188, 1597 (1987).

[16] H. F. Leube and H. Finkelmann, Macromol. Chem. Phys. 192, 1317 (1991). 